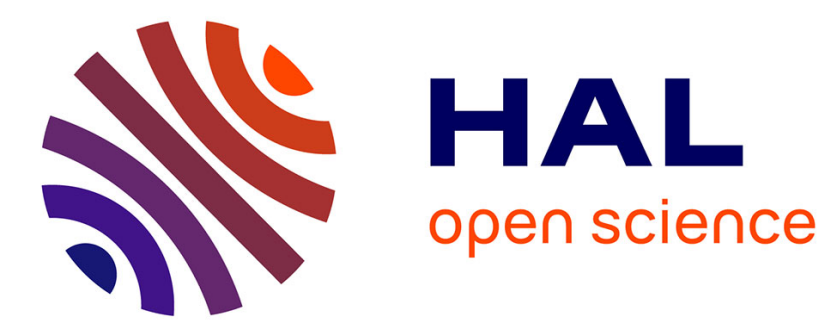

\title{
Experimental evidence of a helical, supercritical instability in pipe flow of shear thinning fluids
}

\author{
L. Picaut, O. Ronsin, C. Caroli, T. Baumberger
}

\section{To cite this version:}

L. Picaut, O. Ronsin, C. Caroli, T. Baumberger. Experimental evidence of a helical, supercritical instability in pipe flow of shear thinning fluids. Physical Review Fluids, 2017, 2 (8), pp.083303. 10.1103/PhysRevFluids.2.083303 . hal-01614727

\section{HAL Id: hal-01614727 https://hal.sorbonne-universite.fr/hal-01614727}

Submitted on 11 Oct 2017

HAL is a multi-disciplinary open access archive for the deposit and dissemination of scientific research documents, whether they are published or not. The documents may come from teaching and research institutions in France or abroad, or from public or private research centers.
L'archive ouverte pluridisciplinaire HAL, est destinée au dépôt et à la diffusion de documents scientifiques de niveau recherche, publiés ou non, émanant des établissements d'enseignement et de recherche français ou étrangers, des laboratoires publics ou privés. 


\title{
Experimental evidence of a helical, supercritical instability in pipe flow of shear thinning fluids.
}

\author{
L. Picaut, O. Ronsin, C. Caroli and T. Baumberger \\ Institut des NanoSciences de Paris, CNRS, Sorbonne Université - Pierre et Marie Curie, \\ UMR 7588, 4 place Jussieu, 75005 Paris, France
}

\begin{abstract}
We study experimentally the flow stability of entangled polymer solutions extruded through glass capillaries. We show that the pipe flow becomes linearly unstable beyond a critical value $\left(\mathrm{Wi}_{c} \simeq 5\right)$ of the Weissenberg number, via a supercritical bifurcation which results in a helical distorsion of the extrudate. We find that the amplitude of the undulation vanishes as the aspect ratio $L / R$ of the capillary tends to zero, and saturates for large $L / R$, indicating that the instability affects the whole pipe flow, rather than the contraction or exit regions. These results, when compared to previous theoretical and experimental works, lead us to argue that the nature of the instability is controlled by the level of shear-thinning of the fluids. In addition, we provide strong hints that the non-linear development of the instabiilty is mitigated, in our system, by the gradual emergence of gross wall slip.
\end{abstract}

\section{INTRODUCTION}

Extrusion, which consists in forcing viscoelastic polymer melts or solutions out of a reservoir through an axisymmetric pipe, or "die", is an important shaping process, unfortunately prone to a variety of instabilities which spoil the surface or the bulk of the extrudate [1-3]. Among these, an ubiquitous one, usually referred to as "melt fracture", results in a distortion of the extrudate into a more or less pronounced and regular helical shape. Visualization of the velocity and stress fields in the reservoir [3-6] has lent strong support to the idea that melt fracture was ascribable to the well documented destabilization of the elongational flow upstream of the sudden contraction at the entry of the die $[4,7]$. Due to viscous damping, such velocity oscillations are expected to decay over a finite length as the fluid is advected through the pipe. Hence, it is not surprising that helical instabilities were reported by many authors as being more severe when using short dies, rather than pipes with larger length/radius $(L / R)$ aspect ratios [3]. However, as pointed out by Larson [1], one cannot rule out that the flow in a long pipe may itself develop a bulk helical instability, in which case the amplitude of the extrudate distorsion should not decrease upon increasing the die aspect ratio.

The stability of the flow in an infinitely long pipe, in the limit of vanishing Reynolds numbers, has been the subject of extensive theoretical studies, in the case of viscoelastic fluids obeying either the Oldroyd-B $[8,9]$ or the White-Metzner $[10,11]$ constitutive equations. In the first case, dealing with the peculiar class of non shear thinning fluids, it has been shown that the Poiseuille flow, although linearly stable, exhibits a weakly non-linear subcritical instability: beyond a critical value of the Weissenberg number Wi, the flow is unstable against a finite but small noise amplitude, therefore resulting in a hysteretic behavior $[8,9]$. The existence of such an instability has later been confirmed by the experimental studies of Bertola et al. [12] Bonn et al. [13] and Pan et al. [14] on axisymmetric pipe and $2 \mathrm{D}$ channel flows.

A wider class of great practical importance deals with polymeric fluids which exhibit both a large first normal stress difference and strong shear thinning in the same range of shear rates. It has been studied theoretically by Wilson and coll. [10, 11, 15] within the framework of the White-Metzner equation, under the assumption of a power law dependence of the shear-rate dependent viscosity $\eta(\dot{\gamma})$. They have shown that the base flow is linearly unstable provided that the shear thinning exponent $n=1-|d \log \eta / d \log \dot{\gamma}|$ is smaller than $0.3[10,11]$. The non-linear behavior has not been investigated, however, so that the super- or subcritical nature of the instability remains unpredicted. Strong hints that a bulk flow instability actually occurs beyond a finite $\mathrm{Wi}$ value in strongly shear-thinning fluids $(n \simeq 0.2)$ have been provided by Bodiguel et al. [16] who observed the emergence of sinuous tracer paths in microfluidic $2 \mathrm{D}$ channels. This emergence is associated with that of strong fluctuations of the velocity field, as confirmed by Poole [17] using larger scale pipe flows with $R e \simeq 100$. However, due to the difficulty of quantifying the spatio-temporal behavior of the velocity field inside the die, these studies did not permit a full characterization of the nature of the bifurcation, in terms of amplitude evolution and possible presence of hysteresis.

Here, we report on a study of the extrusion through long glass capillaries (aspect ratios $L / R$ up to 200) of a strongly shear thinning $(n \simeq 0.3)$ polymer solution exhibiting a rheology well described by a White-Metzner equation. A detailed quantitative analysis of the extrudate shape, together with the observation of tracer paths in the glass capillaries, provide experimental proof of: (i) the existence of an instability associated with the helical distorsion of the extrudate, (ii) the supercritical nature of the bifurcation, which occurs beyond a critical Weissenberg $\mathrm{Wi}_{c} \simeq 4-6$, with a wavelength $\lambda_{c} \simeq 3 R$, (iii) the concomitant emergence of undamped velocity oscillations extending along the whole length of the capillary,

On this basis, we provide what we think is the first 
evidence and characterization of a helical linear instability of the axisymmetric base flow in a long pipe, in the vanishing Reynolds number limit. We are therefore able to conclude that, at least for shear thinning viscoelastic fluids, upstream destabilization of the contraction flow is not the sole route to the helical extrudate instability which, though an ubiquitous phenomenon, has attracted much less attention in the theoretical litterature than the "sharkskin" or "spurt flow" ones [1, 2, 18].

\section{MATERIALS AND METHODS}

\section{A. Polymer solutions: preparation and rheological characterization}

Polymer solutions were prepared by dissolving sodium alginate (average molecular mass $\bar{M}=246 \mathrm{kDa}$, Kalys S.A., France), a biopolymer extracted from seaweed, in deionized water. $500 \mathrm{ppm}$ sodium azide were added to prevent bacterial development. We have made use of solutions with alginate concentration $c=4,6$ or $10 \mathrm{wt} . \%$, referred to in the following as ALG4, ALG6 or ALG10. At the lowest concentration (ALG4), overnight magnetic stirring was sufficient to obtain a homogeneous solution. For higher concentrations, alginate powder was left to swell and dissolve for several days under periodic gentle manual stirring. Homogeneity of the solution was assessed from its optical aspect.

Rheological measurements were performed using a stress-controlled rheometer (MCR 501, Anton Paar) equipped with a $50 \mathrm{~mm}$ diameter, $2^{\circ}$ cone-plate, sandblasted cell geometry.

The concentration dependence of the zero-shear specific viscosity [19][20] yields a concentration at the onset of the entanglement regime $c_{E}=1 \mathrm{wt} . \%$, so that the three systems tested in this work are well entangled polymer solutions. Their viscoelastic properties were characterized by the measurement of both the steady flow viscosity $\eta(\dot{\gamma})$ and the first normal stress-difference $N_{1}(\dot{\gamma})$ for shear rates $\dot{\gamma}$ in the range $10^{-1}-10^{2} \mathrm{~s}^{-1}$.

The measurement of the first normal stress difference is known to be fraught with potential experimental errors [21]. Laun has proposed a procedure, akin to the CoxMerz rule [22], to derive $N_{1}(\dot{\gamma})$ from the linear response moduli $G^{\prime}(\omega)$ and $G^{\prime \prime}(\omega)$. As illustrated in [19] material, we have checked on the ALG10 solution that Laun's empirical rule was accurate enough to legitimate its use with ALG4 and ALG6 solutions, the much lower level of normal forces involved with these solutions preventing reliable direct measurements of $N_{1}$. The data presented on Fig. 1 have been obtained according to this procedure.

As seen on Fig.1.a, the three solutions exhibit a marked shear thinning behavior that we have characterized using a Cross law:

$$
\eta=\frac{\eta_{0}}{1+\left(\dot{\gamma} / \dot{\gamma}_{0}\right)^{1-n}}
$$

Fitting values of the plateau viscosity $\eta_{0}$ and crossover shear rate $\dot{\gamma}_{0}$ are given in Table I. We found that a single shear-thinning exponent $n$ was able to account for the behavior of the three solutions, namely $n=0.29 \pm$ 0.02 , a value compatible with the "universal" one $(n \simeq$ 0.24 ) reported for a broad class of entangled, random coil polysaccharide solutions [23] to which alginate belongs.

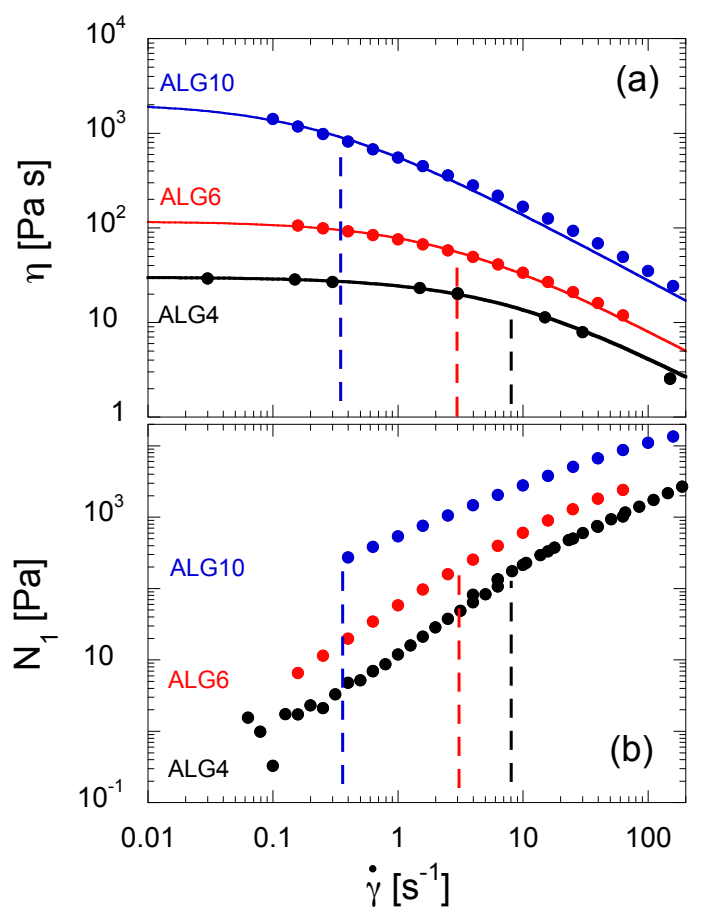

FIG. 1. Rheological characteristics of the shear-thinning alginate solutions. (a) Flow viscosity $\eta$ : The solid lines are a fit by a Cross law with a common shear-thinning exponent $n=0.29$. The dashed lines mark the crossover shear rates $\dot{\gamma}_{0}$. Parameters of the fit are listed in Table I. (b) Fist normal stress difference.

TABLE I. Plateau viscosity $\eta_{0}$ and crossover shear rates $\dot{\gamma}_{0}$ values for the three alginate concentrations $c$ used in this study. The last two columns give the critical values of the average shear rate and the corresponding Weissenberg number estimated at the capillary wall at the onset of the helical instabiliy.

\begin{tabular}{|c|c|c|c|c|c|}
\hline & $c$ [wt.\% $]$ & $\eta_{0}[\mathrm{~Pa} \mathrm{~s}]$ & $\dot{\gamma}_{0}\left[\mathrm{~s}^{-1}\right]$ & $V /\left.R\right|_{c}\left[\mathrm{~s}^{-1}\right]$ & $\mathrm{Wi}_{c}$ \\
\hline ALG4 & 4 & 30 & 8 & $340-500$ & $5-5.6$ \\
ALG6 & 6 & 110 & 3 & 60 & 4 \\
ALG10 & 10 & 1900 & 0.3 & $8-14$ & $4.3-5.1$ \\
\hline
\end{tabular}

Fig. 2 shows the shear rate dependence of the Weissenberg number Wi defined as:

$$
\mathrm{Wi}=\frac{N_{1}(\dot{\gamma})}{\eta(\dot{\gamma}) \dot{\gamma}}
$$


As commonly observed with shear-thinning viscoelastic fluids [16] Wi $(\dot{\gamma})$ exhibits a markedly sublinear growth indicative of an extended spectrum of viscoelastic relaxation times. This is reasonably well described for $\dot{\gamma}>\dot{\gamma}_{0}$ by a simple power law:

$$
\mathrm{Wi} \simeq\left(\dot{\gamma} / \dot{\gamma}_{0}\right)^{m}
$$

with $m \simeq 0.3$. From $m \simeq n$, we conclude that entangled alginate solutions are well described by a White-Metzner model, yielding $\eta=G \tau$ and $N_{1}=2 G(\dot{\gamma} \tau)^{2}$, with a constant elastic modulus $G$ and a shear-rate dependent relaxation time $\tau \sim \dot{\gamma}^{n}$ for $\dot{\gamma}>\dot{\gamma}_{0}$.

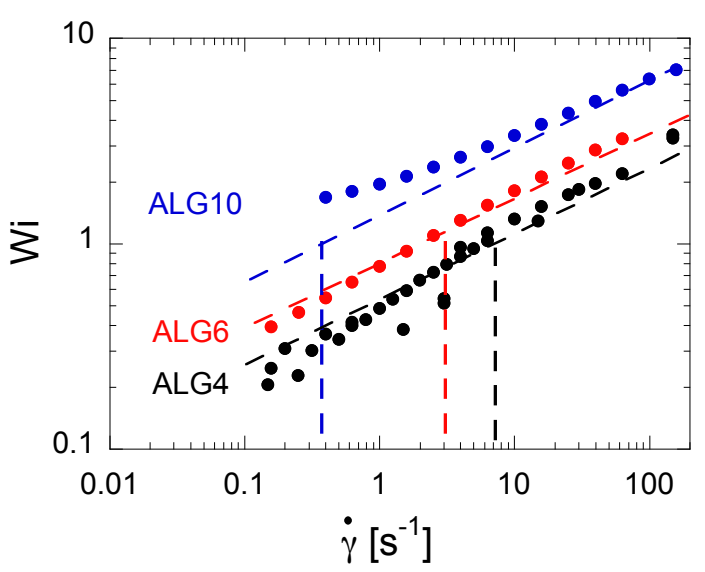

FIG. 2. Sublinear, shear rate dependent Weissenberg number for alginate solutions. The dashed lines indicates the values of $\dot{\gamma}_{0}$ and the full lines are $\left(\dot{\gamma} / \dot{\gamma}_{0}\right)^{m}$ with $m \simeq 0.3$.

\section{B. Extrusion set-up}

Polymer solutions were extruded through cylindrical capillaries of inner radius $R$ and length $L$ using commercial $1 \mathrm{~mL}$ syringes. The body of the syringe was filled with the viscous polymer solution using a spatula. The volumetric throughput $\mathcal{Q}$ was prescribed by driving the piston velocity through a home-made syringe pump operated by a stepping motor. As usual when working with microfluidic circuits, their finite compressibility associated with their large hydraulic impedance results in long transients. These were monitored by measuring the force on the piston with an on-line transducer. Fortunately, thanks to shear thinning, the impedance decreases markedly as the throughput increases so that for all $\mathcal{Q}$ values used in this study, the volume of fluid extruded during a transient remained negligibly small. In the following, we will characterize the steady state flow by the average velocity in the capillary $V=\mathcal{Q} /\left(\pi R^{2}\right)$.

Except when specified, we have made use of cylindrical borosilicate glass capillaries with radii 100, 150 and 250

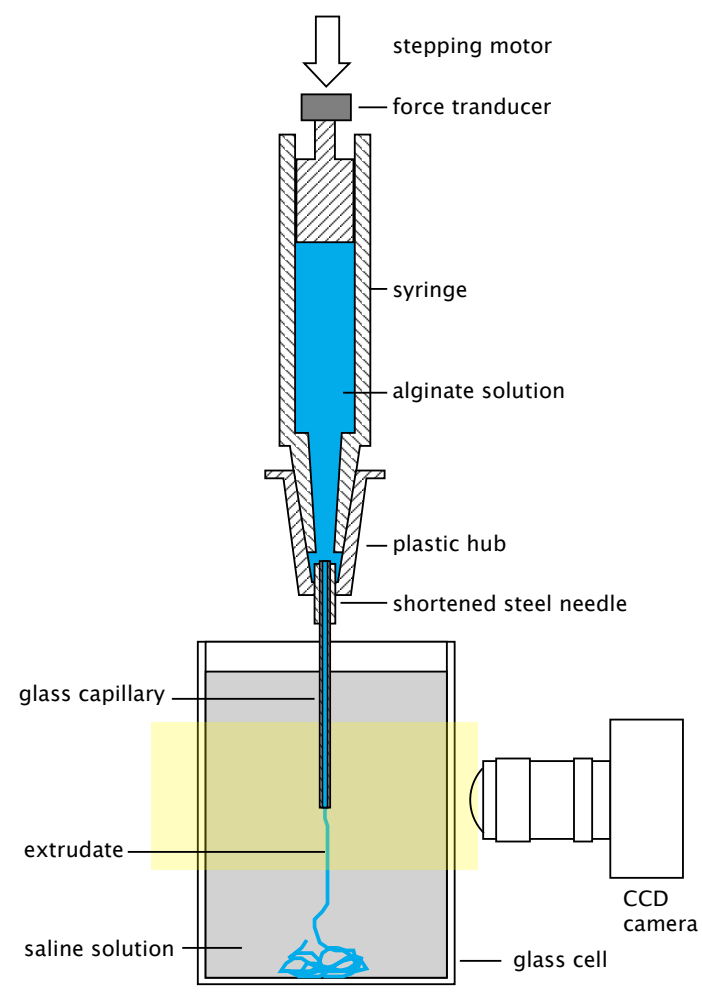

FIG. 3. Schematic representation of the extrusion setup.

$\mu \mathrm{m} \pm 10 \%$ (VitroCom, USA). Each capillary, used as purchased, was first diamond-cut to the prescribed length, then inserted into a shortened commercial steel needle, with an inner radius chosen to tightly fit the outer radius of the capillary. The assembly was finally epoxy-glued together, with the capillary end just emerging out of the steel needle, into the tappered plastic hub (see Fig. 3). The exit end of the syringe has an outer (resp. inner) diameter of 4 (resp. 1) $\mathrm{mm}$.

In order to minimize surface tension and gravityinduced drawing effects on the extrudate, extrusion was carried out into a bath of aqueous liquid. We have made use of a $0.5 \mathrm{M} \mathrm{MgCl}_{2}$ saline solution into which alginate is poorly soluble so that, although $\mathrm{Mg}^{2+}$ ions do not crosslink alginate molecules, the extrudate preserves its shape and sharp interface long enough for its distorsions to be quantified.

The whole set-up (syringe, capillary and bath) was thermostated at $T=20 \pm 0.5^{\circ} \mathrm{C}$.

The extrusion bath being contained in a glass cell, significant lengths (typically $\simeq 20 R$ ) of the capillary and the extrudate on either side of the exit plane can be monitored under direct, quasi-parallel illumination, using a video camera equipped with a macro objective and operating at speeds up to $300 \mathrm{frame} / \mathrm{s}$. Sequences where acquired for each value of the control parameter $V$ and stored for analysis. 


\section{Profile analysis}

The undulation amplitude $\Delta R$ of the extrudate (see left panel Fig.4) is measured from the reconstruction of its profile, using the section visible at each time $t$ within the field of view. First of all, we select one edge and determine its position $y(t)$ at a distance $x=D$ from the exit plane of the capillary (see Fig. 4 for the definition of $x$ and $y)$. A value $D=4 R$ is chosen so as to be out of the zone of viscoelastic recovery responsible for the "die swelling" phenomenon. The edge position in the fixed framework linked to the capillary evolves with time due to the advection of the distorded extrudate along the capillary axis but also perpendicular to it due e.g. to the flagellar motion associated with the sinuous distorsion. The latter contribution is redundant and must be removed. We therefore determine the translation vector of the extrudate at time $t$ in the vicinity of the abscissa $D$ by applying a standard image correlation technique in a zone of interest between two successive frames. The $y$-component is used to correct the edge position while the $x$-one is used to map the time $t$ onto the position $x$ along the capillary axis. We thus obtain the shape $R(x)$ of the profile edge corresponding to an extrudate length $\simeq 300 R$. It is Fourier transformed so as to get the power spectrum from which a peak is identified, centered on a spatial frequency $f_{0}$ (Fig.5). Finally, from the area of the peak, one computes the r.m.s. amplitude of the corresponding oscillations, named $\Delta R$ in the following.

\section{EXPERIMENTAL RESULTS}

The three solutions under study exhibit the same qualitative behavior when extruded at increasing values of $V$. Except when explicitly stated, the data presented in this section have been obtained with ALG10 solutions.

\section{A. Characteristics of the instability}

The following description refers to the labelled pictures on the left-side panel of Fig.4.

At very low throughput (a), the extrudate profile is cylindrical and smooth. Then (b), upon increasing $V$, although the extrudate remains straight, a characteristic fine pattern appears on its surface. The pattern contrast gradually increases with $V$, indicating the growth of the surface distorsion until it shows up as a sawtooth deformation of the profile edges (c), unmistakably attributable to the "sharkskin" instability [1-3].

In the vicinity of a velocity $V_{c}$, the extrudate profile, as well as trains of micro bubbles which are sporadically coextruded, exhibit bursts of wavy distorsion (c). When increasing $V$ slightly above $V_{c}$, a wavy instability clearly develops. It manifests itself as a fairly regular, sinuous undulation of the profile edges (d). It is worth noting that the sharkskin pattern does not vanish at the onset of the larger scale undulations but remains superimposed to them, which suggests that both instabilities have independent physical and spatial origins.

The amplitude of the undulation increases rather sharply upon increasing $V$ above $V_{c}$ and reaches a plateau for $V / V_{c} \simeq 1.2(\mathrm{~d})$, (e). Finally, we have observed no further qualitative changes in the extrudate aspect and/or dynamics upon further increasing $V / V_{c}$ up to typically 10 , i.e. the undulations remain regular and no other instability occurs.

In the following we focus on this instability — leaving the sharkskin one aside - and refer to it as "the" instability. In order to put this description on a more quantitative basis, we have systematically measured the rms. amplitude of the oscillations $\Delta R$ and their mean spatial frequency $f_{0}$, when the undulating mode was present.

a. The instability is helical - In order to assess the full $3 \mathrm{D}$ symmetry of the extrudate undulation, we have measured $\Delta R$ at a single velocity $V \gtrsim V_{c}$, for a fixed position of the camera but different values of the azimuthal angle $\theta$ around the capillary axis. Fig. 6 shows that the undulation amplitude is $\theta$-independant. This, together with the sinuosity of the profile, demonstrates that the extrudate is deformed into a helical shape.

b. The instability is supercritical - A possible subcritical nature of the instability would be revealed by the occurence of hysteresis in the oscillation amplitude when ramping $V$ up and down [12]. Fig.7 shows that, within experimental uncertainties, no hysteresis is measurable, either on the amplitude or on the spatial frequency of the undulations. We can therefore conclude that the instability occurs via a supercritical bifurcation. This is compatible with the existence of damped bursts of undulation close below the threshold. Accordingly, we determine the critical velocity $V_{c}$ by extrapolating to $\Delta R=0$ the universal behavior of the amplitude in the close vicinity of the threshold for a supercritical (Hopf) bifurcation [24], namely $(\Delta R)^{2} \sim V-V_{c}$.

As $V / V_{c}$ grows, the amplitude $\Delta R$ departs from its universal behavior and tends to plateau at a value $\Delta R_{\max }$ which remains relatively small, namely $\Delta R_{\max } / R \lesssim 0.1$. Accordingly, the profile edges remain quasi-sinusoidal (ie. the non-linear terms of the underlying dynamics remain small). More precisely, the power spectra of $R(x)$ show no harmonic peak and remain relatively narrow (typ. $\left.\Delta f / f_{0} \simeq 0.2\right)$ in the whole range $\left(V / V_{c}<10\right)$ investigated. The spectrum shown on Fig.5, which corresponds to $V / V_{c} \simeq 1.2$, i.e. to the maximum $\Delta R_{\max }$ of oscillation amplitude, is therefore typical.

c. The instability is controlled by the Weissenberg number - Fig.8 shows the variations of $\Delta R$ and $f_{0}$ with $V$ for ALG10 flowing through capillaries with three different radii $R$. Clearly, the critical velocity $V_{c}$, the maximum undulation amplitude $\Delta R_{\max }$ and the spatial frequency $f_{0}$ depend on the capillary radius. Fig.9 shows that $V_{c}, \Delta R_{\max }$ and the pitch of the helical disturbance $\lambda=1 / f_{0}$ depend linearly on $R$.

Accordingly we have replotted the data, suitably scaled 


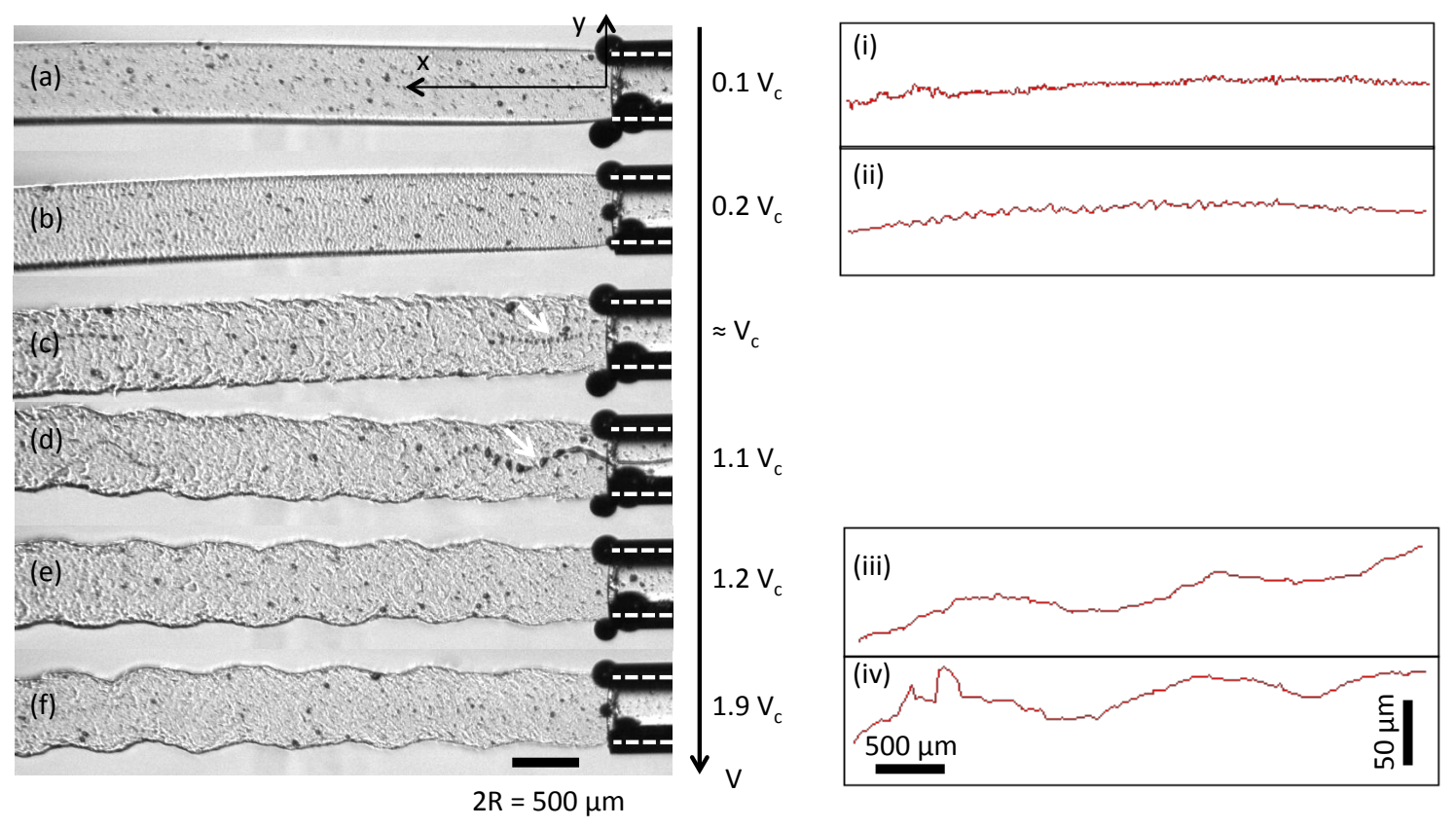

FIG. 4. Left pannel: Snapshots of the extrudate exiting from a glass capillary with internal radius $R=250 \mu \mathrm{m}$ (see dashed white lines) for increasing average velocities $V$. At a critical value $V_{c}$ sinuous features show up, such as trains of microbubbles arranged sinusoidally, indicated by white arrows on (c) and (d), see text. The slight bending of the extrudate visible on (b-c) is due to the large scale viscous coiling of the extrudate from the bottom of the glas cell. Right pannel: Characteristic paths of microbubbles tracked in the end portion of the capillary extending $\simeq 20 R$ upstream of the exit plane. The velocity scale is common to both panels. We have expanded the vertical scale in order to magnify the bubble displacement perpendicular to the axis.

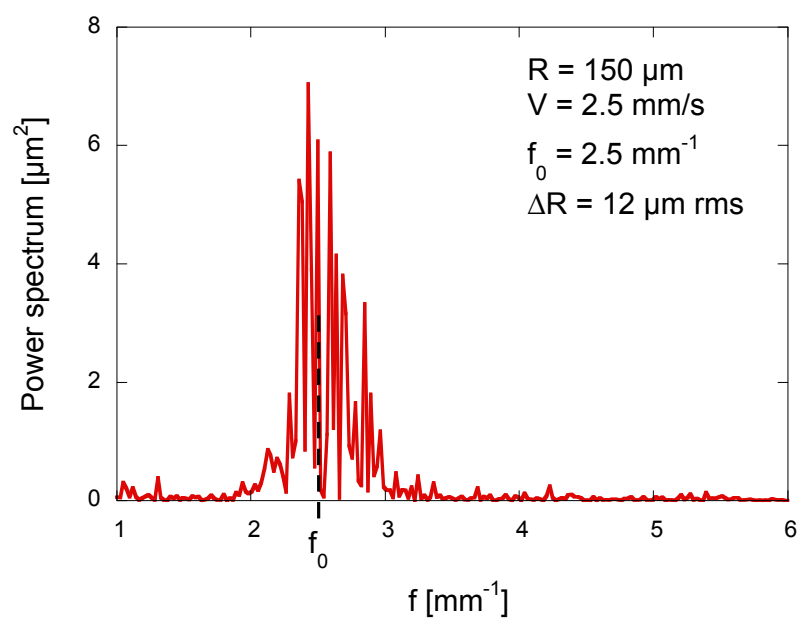

FIG. 5. Power spectrum of a sinuous extrudate profile $R(x)$ for a velocity $V \simeq 1.2 \times V_{c}$ with $V_{c}$ the velocity at the onset of oscillations (see text).

by $R$ (Fig.10). For a given alginate concentration (ALG10) the relevant control parameter is therefore the average shear-rate $V / R$. We have determined the critical value $V /\left.R\right|_{c}$ for ALG4 and ALG6 as well. The values compiled in Table I are compatible with a critical value

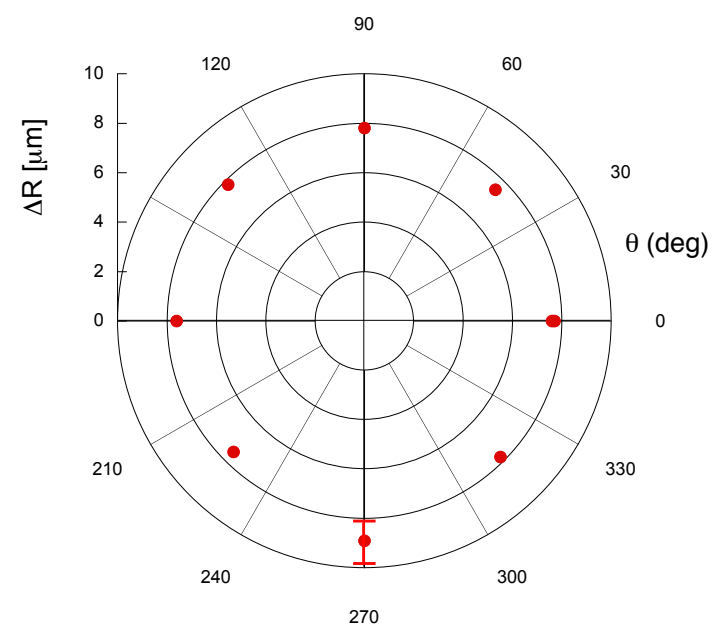

FIG. 6. Azimuthal isotropy of the rms amplitude of the extrudate undulation $\left(V=2 \times V_{c}\right)$ as measured by a fixed camera but for different angular positions $\theta$ of the whole extrusion setup $(R=100 \mu \mathrm{m})$ around its symmetry axis.

$\mathrm{Wi}_{c}=4-6$ of the Weissenberg number (evaluated at the wall, under a no-slip hypothesis, as detailed in [19]). 


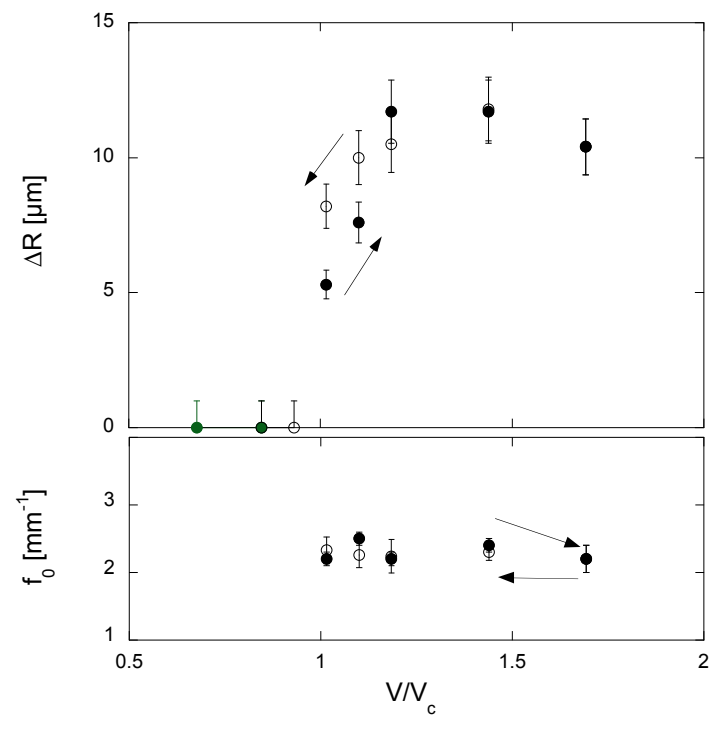

FIG. 7. Evolution of the oscillation amplitude and spatial frequency upon first increasing, then decreasing the control parameter $V$ in the vicinity of the threshold $V_{c}$, for a capillary with $R=150 \mu \mathrm{m}$, showing no measurable hysteresis.

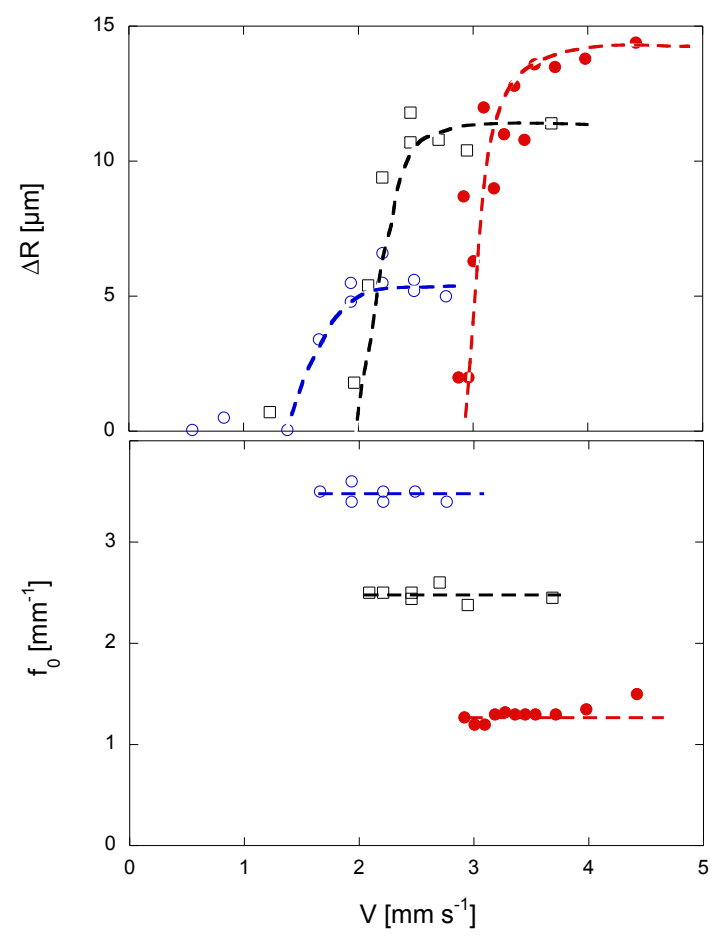

FIG. 8. Evolution of the oscillation amplitudes and spatial frequencies $R=100 \mu \mathrm{m}$ (red full circles), $150 \mu \mathrm{m}$ (black squares) and $250 \mu \mathrm{m}$ (blue open circles). The dotted lines are guides for the eyes.

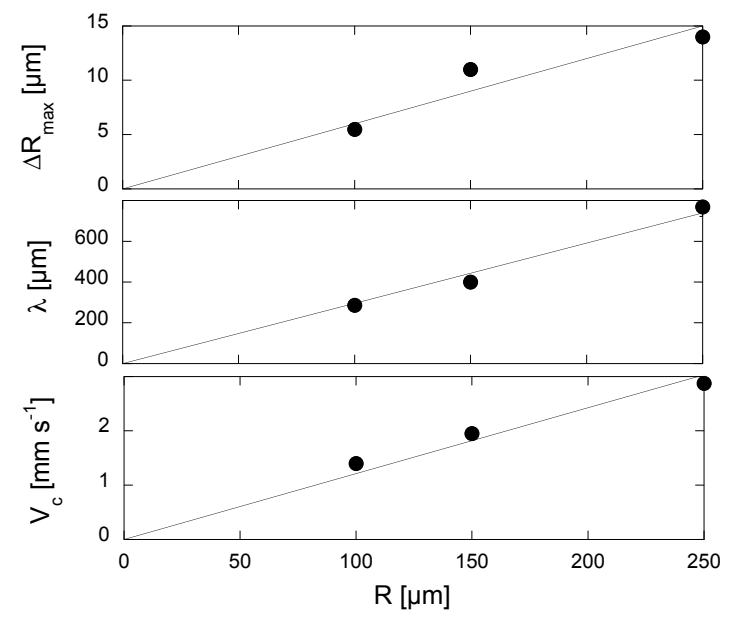

FIG. 9. Amplitude $\Delta R_{\max }$ at apparent saturation, wavelength $\lambda=V_{c} / f_{0}$ of the oscillations at the onset of the oscillations, and threshold velocity $V_{c}$ with respect to the capillary radius $R$.

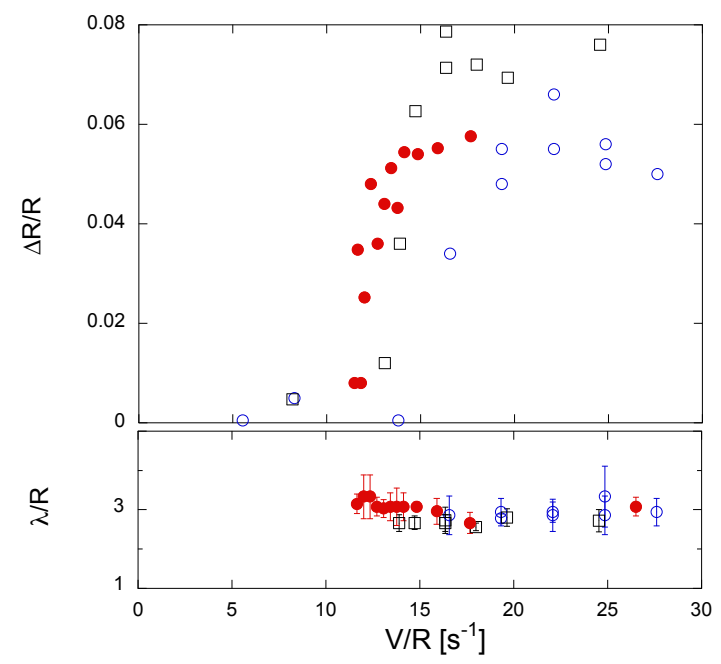

FIG. 10. Characteristics of the instability in the vicinity of its threshold, plotted in reduced form. Same symbols as on Fig.8

\section{B. Evidence of a bulk flow instability inside the capillary}

Oscillations of the extrudate necessarily result from an oscillating velocity field inside the die, at least in the close vicinity $(\simeq R)$ of the exit plane. In order to check this, we have tracked microbubbles advected along the glass capillary in the end region extending $\simeq 20 R$ upstream of the exit plane. The right panel of Fig.4 shows representative paths selected at different average velocities below and above the threshold $V_{c}$. As expected, whereas for $V<V_{c}$ the paths are straight, for $V>V_{c}$ their projec- 
tion onto the observation plane are quasi-sinusoidal.

At this stage, we must assess whether the flow instability responsible for the extrudate one is an entry, exit or bulk one. For this purpose, we have monitored for $V>V_{c}$ bubble paths at different locations along the capillary, up to a distance $\simeq 80 R$ upstream of the exit. The observation of quasi-sinusoidal paths with comparable amplitudes and periods, whatever their location along $x$, illustrated on Fig. 11, indicates the presence of undamped oscillations of the velocity field, i.e. an instability which, in contradistinction to the sharkskin one [1], is not localized in the vicinity $(\simeq R)$ of the exit plane.

Moreover, note that the wavelengths of the bubble paths are $\simeq 8 R$, i.e. significantly larger than the period $(\simeq 3 R)$ of the extrudate distorsion. This was already pointed out by Wilson and Loridan [11] in their analysis of Bodiguel et al. data [16]. Such an apparent wavelength dilatation is akin to a Doppler effect. The analysis performed in [19] enables us to estimate that the magnitude of the phase velocity $v_{\varphi}$ of the oscillating perturbation is comparable with $V$.

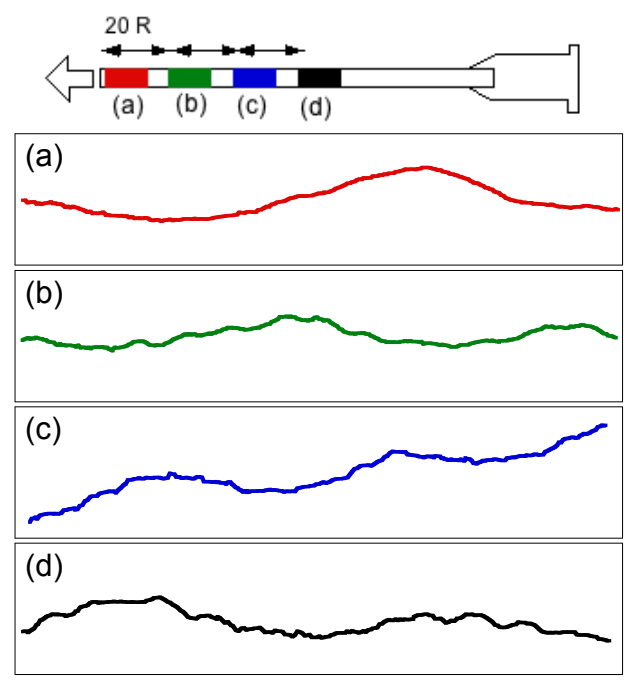

FIG. 11. Paths of lagrangian tracers recorded at different locations along a $R=250 \mu \mathrm{m}$ capillary. The portions of the capillary corresponding to each path are shown on the upper drawing.

Finally, the fact that the extrudate instability is observed for capillaries with aspects ratios $L / R$ as large as 200 strongly suggests that it cannot originate from a destabilization of the flow upstream of the abrupt contraction. As pointed out by Larson [1], some unstable pipe modes must be at work in order to pump energy from the base flow and compensate for the viscous losses associated with the velocity oscillations.

In order to make a more precise statement, we have constructed capillaries with a $5: 3$ contraction by glueing a $R_{-}=150 \mu \mathrm{m}$ capillary inside a $R_{+}=250 \mu \mathrm{m}$ one and cutting the narrow portion at different lengths $L_{150}$ (see inset of Fig.12). The throughput range was chosen so that the flow in the wider capillary remained stable while enabling to cross the instability threshold - as revealed by the undulations of the extrudate - in the narrower one. Fig.12 shows the maximum amplitude $\Delta R_{\max }$ of the undulations as a function of $L_{150} / R_{-}$. At large enough aspect ratios, i.e. $L_{150} / R_{-} \gtrsim 40, \Delta R_{\max }$ becomes insensitive to the length. This definitely establishes that the helical deformation of the extrudate is the outer manifestation of an instability which affects the whole flow inside the capillary. The results presented so far were obtained for $L / R \simeq 200$, hence correspond to the instability of an "infinite" pipe. For $L / R<40$, the amplitude decreases markedly and, when extrapolated to the "orifice die" limit $(L / R \rightarrow 0)$ the amplitude vanishes (Fig.12). As will be discussed in the next section, this is in strong contradistinction with the usually reported phenomenology for polymer melt extrusion where shorter dies, mostly used in practice, are also the most prone to the so-called "melt fracture" helical instability.

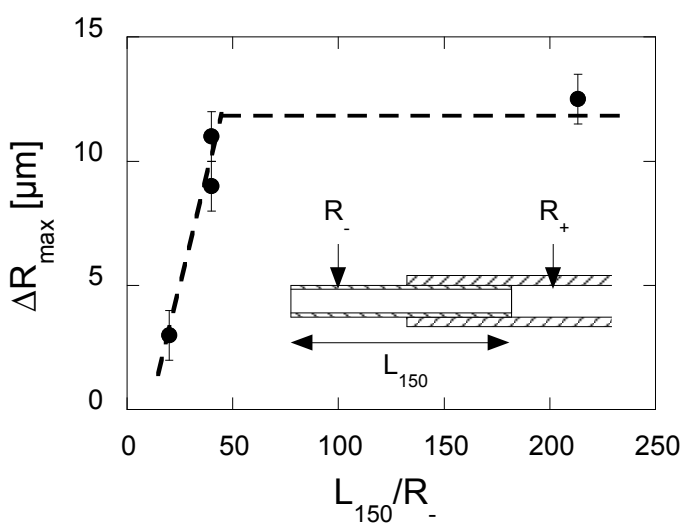

FIG. 12. Maximal amplitude of undulation of a extrudate exiting a $R_{-}=150 \mu \mathrm{m}$ capillary at a distance $L_{150}$ downstream a 5:3 contraction with $R_{+}=250 \mu \mathrm{m}$ (see sketch on inset). The broken lines are guides for the eyes.

\section{DISCUSSION}

\section{A. Comparison with previous studies}

d. Influence of shear-thinning on the nature of the instability - The results presented so far are in clear contradistinction with those of Bertola et al. [12] and Pan et al. [14] who evidenced a pipe instability controlled by the Weissenberg number but exhibiting a large hysteresis - an obvious subcritical feature, while the instability is, in our case, supercritical. Since the two cases differ essentially by the steady flow characteristics of the fluids under study, namely their being shear-thinning or not, we are led to conclude that it is this rheological feature which primarily controls the nature of the pipe instability. This is fully supported by the theoretical predic- 
tion that planar channel flows of "weakly" shear-thinning White-Metzner fluids $(n>0.3)$ are linearly stable [10] while "strongly" shear-thinning ones $(n \leq 0.3)$ are linearly unstable. The theoretical limiting value of $n$ for axisymmetric pipe flows is unknown but our results indicates that, in this respect, entangled alginate solutions with $n \simeq 0.3$ belong to the "strongly shear thinning" class of fluids.

Beyond proving the existence of a linear pipe flow instability, our results reveal that: (i) it corresponds to a direct Hopf bifurcation - a feature which would require a non-linear analysis of the flow dynamics to be theoretically assessed - and (ii) the first unstable mode is helical - hence a relevant theoretical study of the flow stability should be fully $3 D$ [18].

As far as symmetry breaking is concerned, our pipe flow helical instability is strongly reminiscent of the sinuous one reported by Bodiguel et al. [16] for 2D microchannel flows. Although the high molecular weight, partially hydrolyzed polyacrylamide (PAa) solutions used in their study exhibited an exponent $n \simeq 0.2$, i.e. were highly shear thinning, the results could not be directly compared to the theoretical predictions of [10]. Indeed, on the one hand, for technical reasons [15], the theoretical flow stability was restricted to "varicose" axisymmmetric modes. On the other hand, PAa does not obey the White-Metzner constitutive equation with a single exponent ruling power laws (1) and (3). In [16], $n=0.21$ and $m=0.43$ indicates that the elastic modulus itself is rate dependent. The consequence of this extra complication has been studied by Wilson \& Loridan [11] using an extended constitutive law. Interestingly, albeit they have restrained their analysis to varicose modes, they have shown that the flow stability, i.e. the critical value of the Weissenberg number, is noticeably increased by the rate dependence of the modulus. This is an important example showing that rheological details may have quantitative consequences on a flow instability while preserving its qualitative characteristics. This would be worth being confirmed experimentally by swapping the two fluid systems - ALG and PAa - in each of the two flow configurations of [16] (2D channel flow) and the present study (axisymmetric pipe flow).

e. Competition between pipe and contraction flow instabilities - In the field of extrusion instabilities, a touchstone is the broad phenomenology pertaining to the so-called "melt fracture" of polymer melts $[3,5,25]$. In their early review, Petrie and Denn [26] pointed out the apparent contradiction between two groups of experimental results dealing with either branched or linear polyethylene. For branched, low density polymer melts, the severity of extrudate distorsion does decrease as the die length is increased, while the opposite effect is observed for linear, high density polyethylene. Despite of its intriguing nature, clearly suggesting two distinct types of instabilities, this phenomenon has not been studied systematically until recently. Here we focus on the extensive study by Combeaud et al. [6, 25] of the helical extru- sion instability of linear polystyrene (PS). Their polymer melts and our entangled polymer solutions exhibit a common rheological characteristic since the flow viscosity of PS is strongly shear thinning [25] with a power law exponent $n \simeq 0.2$, a plateau viscosity $\eta_{0} \simeq 5 \times 10^{4} \mathrm{~Pa}$.s and a cross-over shear rate $\dot{\gamma}_{0} \simeq 1 \mathrm{~s}^{-1}$. It appears therefore meaningful to compare the results obtained on PS and ALG, taking advantage that in both studies, the extrudate together with the upstream (pipe or contraction) flow have been characterized.

At first sight, the two systems behave similarly since in both cases the extrudate exhibits at large enough throughput a transition from a cylindrical to a fairly regular helicoidal shape. The authors report that for PS this instability occurs at shear rates far beyond the range accessible in a cone-plate rheometer. Therefore, the critical value $\mathrm{Wi}_{c}$ of the Weissenberg could only be roughly estimated by extrapolation of their rheological data to range between 2 and 10. Since our own estimate is $\mathrm{Wi}_{c}=5 \pm 1$, the comparison is not conclusive, even though both ranges are compatible.

Much more specific is the behavior of the pitch $\lambda$ of the distorted extrudate upon increasing the shear rate. In this respect, both systems show antagonistic trends: while for ALG it is the spatial frequency $\lambda^{-1}$ which remains constant (Fig.8), for PS it is the temporal one $V / \lambda$ which exhibits but small variations. This strongly suggests that both systems develop instabilities of different origins. This is confirmed in [6] by the direct visualization of the velocity field in the reservoir, upstream of the inlet, which clearly indicates that emergence of the extrudate instability coincides with the destabilization of the contraction flow, a phenomenon which has been extensively studied by McKinley et al. for non shear-thinning Boger fluids [7]. Correlatively, as the die aspect ratio is reduced, the extrudate undulation becomes more pronounced for PS, whereas it vanishes for ALG (Fig.12).

Although for practical reasons extrusion of polymer melts is usually performed through short dies, Combeaud et al have extended their study to aspect ratios up to $L / R=64$. They show that the helicoidal undulations persist up to this aspect ratio value, although strongly reduced (see Fig. 3.7 of [25]). The gradual attenuation of the helix amplitude however strongly slow down for $L / R \gtrsim 32$ (see Fig. 3.8.b. of [25]). Interestingly, this value, which could be considered a priori quite large for an "infinite die" regime to be reached, is compatible with our own finding that the "infinite pipe" limit is reached for $L / R \simeq 40$ (12). From this comparison we conclude that the main body of experiments on PS (resp. ALG) are concerned with "short" (resp. "long") dies in which reservoir (resp. pipe) flow instabilities are favored.

In light of the present results, it should be extremely useful to focus experimentally on a contraction flow instability of ALG and a possible pipe flow instability of PS, although the high viscosity of the melts probably precludes using the long pipes required for this instability to fully develop. 


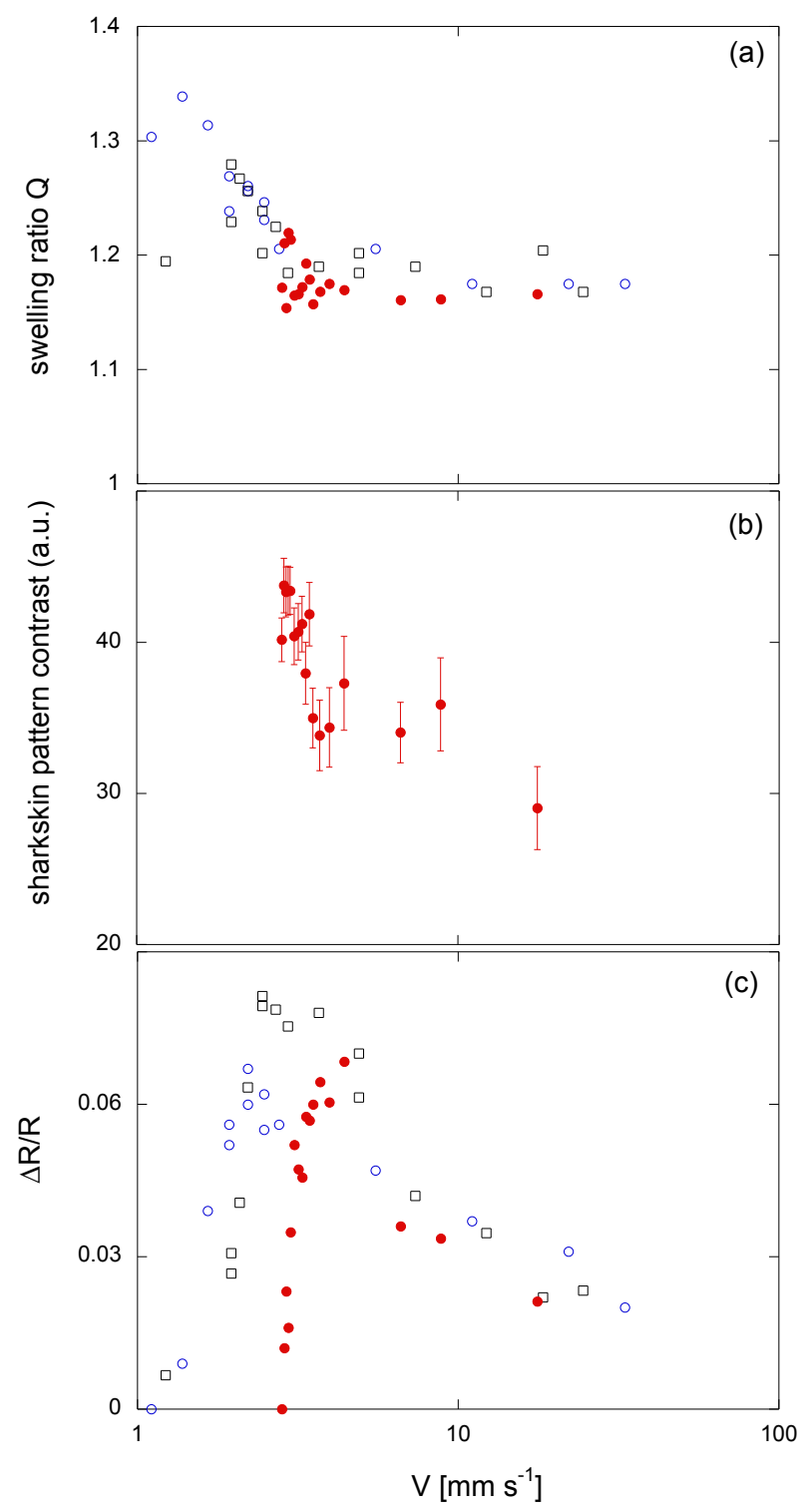

FIG. 13. Evolution with respect to $V$ of (a) the swelling ratio $Q$, (b) the sharkskin contrast index (see text) and (c) the scaled oscillation amplitude. Different radii are represented by the same symbols as in Fig.8.

\section{B. Non-linear instability development vs. wall slip}

The standard extrusion phenomenology of polymer melts involves the gradual transition from a quasiperiodic helical disturbance to a more or less chaotic one as the throughput is increased beyond a critical threshold. With this in mind we have systematically explored the evolution of the extrudate undulations beyond $\mathrm{Wi}_{c}$. No widening of the oscillation spectrum (see Fig.5) was observed up to the experimental limit of $V \simeq 10 \times V_{c}$ (however only a two-fold increase in the slowly varying Wi). Surprisingly, although one could expect a slow- ing down of the amplitude growth due to the build-up of stabilizing non-linear terms in the flow dynamics, we systematically observe a marked decrease of the amplitude, as shown on Fig.13.c. This means that the plateauing trend observed close above $V_{c}$ (Fig.8) signals, in fact, the existence of a maximum of the amplitude. Such a behavior is, to our knowledge, quite unusual in non-linear dynamics.

Since the extrudate undulations involve some motion of the surrounding bath fluid, we have checked whether viscous damping could be involved. We have changed the saline solution (viscosity $\eta_{s} \simeq 10^{-3}$ Pa.s) for a 1 wt. $\%$ solution of sodium alginate (viscosity $\eta_{1 \%} \simeq 10^{-1} \mathrm{~Pa} . \mathrm{s}$ ). No significant difference in the $\Delta R(V)$ curve was observed, neither close to onset nor well beyond threshold, suggesting that the viscosity of the bath is not relevant to the undulation development.

Some further insight into the underlying physical mecanisms responsible for this behavior can be gained from the simultaneous measurement of two characteristics of the extrudate, namely the (die-)swelling ratio $Q$ and the sharkskin amplitude. The swelling ratio in the presence of oscillations is determined by measuring the average axial velocity of the extrudate $V_{\text {out }}$ well downstream the capillary exit. The amplitude of the extrudate undulation remains smaller than 0.1 so that the volumetric flow of the extrudate can be approximated to $V \times\left(\pi R_{\text {out }}^{2}\right)$ with $R_{\text {out }}$ the radius of the thread, to within a few percent. Hence we define the die swell ratio as $Q=\left(V / V_{\text {out }}\right)^{1 / 2}$. The sharkskin amplitude is estimated from the contrast of the resulting pattern, determined under constant illumination and video acquisition parameters.

Fig.13.a reveals an unusual behavior of the swelling ratio which is expected to grow as some power law of the shear rate with a small exponent [27]. Here, $Q$ first increases with the throughput but hardly builds up to values larger than 1.3 and then decreases to a plateau value $\lesssim 1.2$ (close to the value 1.1 for a purely newtonian fluid). The same trend is exhibited by the sharkskin contrast (see Fig.13.b and Fig.4): although always superimposed to the volume oscillations, the sharkskin is less and less severe as the throughput is increased beyond threshold.

Both die swell and sharkskin are qualitative indicators of the validity of the no-slip condition at the capillary wall. Die swell is a manifestation of the sudden relaxation of the first normal stress difference which itself requires viscous shear to build up. It is therefore mitigated by wall slip. Sharkskin results from the stress singularity which develops at the exit rim where boundary conditions abruptly switch from no-slip to no-stress [28]. It is attributed to the accomodation of the singularity via tearing of the viscoelastic material [29]. Wall slip clearly smoothes out the singularity hence limits the development of sharkskin. This suggests that the strong attenuation of $\Delta R$ is due to the concomitant build up of wall slip. 
Furthermore, the data of Fig.13.a show that, quite unexpectedly, the relevant variable when the capillary radius is changed is not the shear rate but rather the average velocity $V$. This can be understood as a hint for the occurence of gross slip with a velocity $V_{s} \sim V$. The no slip condition is then replaced by a tribological relationship $\sigma_{w}=f\left(V_{s}\right)$ which depends on "microscopic" characteristics of the fluid-wall interface, and not on the "macroscopic" radius. So, the residual shear rate at the wall $\dot{\gamma}_{w}\left(V_{s}\right) \sim \sigma_{w}^{1 / n}$, must be determined by $V_{s}$, hence by $V$ rather than $V / R$. Accordingly, we have plotted the relative oscillation amplitudes for different radii as a function of $V$ (Fig.13.c). It is clear that, although in the growing part of the amplitude development this variable is not the relevant one, in the decreasing part it leads to a reasonable collapse of the data.

These results, though preliminary, indicate that the non-linear development of the instability is probably mitigated by the gradual build-up of wall slip. We cannot conclude at this point whether the fact that both phenomena occur at neighbouring values of the wall shear rate is a mere coincidence or signals some correlation. Interestingly, non-trivial coupling between flow instability and wall slip can be found in the previously discussed study of Bodiguel et al. [16] (their Fig.2) although the authors have rather focussed on the drag reduction associated with the occurence of elastic turbulence in their microfluidic channel flow.

\section{CONCLUSION}

We have studied in detail the helical instability which occurs when extruding an entangled polymer solution through glass capillaries with large aspect ratios. Coupling the quantitative analysis of the extrudate undulations with the visualization of tracer paths inside the capillaries we were able to demonstrate that this extrusion instability is the downstream manifestation of the destabilization of the base flow occuring everywhere in- side the pipe. The instability exhibits the characteristics of a supercritical Hopf bifurcation occuring above a critical Weissenberg number $\mathrm{Wi}_{c}=5 \pm 1$.

As suggested by the seminal theoretical work of Wilson \& Rallison [10], a key rheological characteristic of our fluid system, as regards its flow stability, is certainly its rather strong shear-thinning behavior. Recent studies have also brought out the essential role played by shear thinning in the stability of viscoelastic flows at zero [30] or moderate [31] Reynolds numbers. In serpentine micro-channels, shear thinning is found to stabilize the flow against the onset of elastic turbulence [30]. In large pipes, it yields an new type of symmetry-breaking supercritical transition [31]. In our case, as well as probably in microchannel planar flow [16], "strong" shear-thinning is responsible for the linear instability of the base flow, whereas Boger fluids designed to exhibit negligible shearthinning are only non-linearly unstable [12-14].

The present work therefore suggests to make use of fluid systems with increasing shear-thinning exponents $n$ in order to study experimentally the evolution of the pipe flow instability, from weakly non-linear subcritical at small $n$ to linear supercritical at larger ones.

An intriguing issue that the present work leaves open is the origin of the large aspect ratio $(L / R \simeq 40)$ required for an "infinite" pipe regime to be reached. To our knowledge, it is the first time that this phenomenon is put forward and we hope that it will stimulate further theoretical and numerical studies.

Finally, preliminary results show that, at least for glass capillaries, the non-linear development of the helical undulations is mitigated by the gradual build-up of wall slip. In the case studied here, the helical instability and the onset of gross slip occur in a narrow bracket of control parameter, leaving open the possibility of some causal relationship. Our set-up makes it possible to change the die material from glass to metal or even PTFE, and will therefore be suitable for future investigation of the role of wall slip in pipe flow instabilities.
[1] Ronald G Larson, "Instabilities in viscoelastic flows," Rheologica Acta 31, 213-263 (1992).

[2] Morton M Denn, "Extrusion instabilities and wall slip," Annual Review of Fluid Mechanics 33, 265-287 (2001).

[3] J-F Agassant, Dawn R Arda, Christelle Combeaud, Armin Merten, Helmut Muenstedt, Malcolm R Mackley, Laurent Robert, and Bruno Vergnes, "Polymer processing extrusion instabilities and methods for their elimination or minimisation," International Polymer Processing 21, 239-255 (2006).

[4] H Nguyen and DV Boger, "The kinematics and stability of die entry flows," Journal of non-Newtonian fluid mechanics 5, 353-368 (1979).

[5] JM Piau, N El Kissi, and B Tremblay, "Influence of upstream instabilities and wall slip on melt fracture and sharkskin phenomena during silicones extrusion through orifice dies," Journal of non-newtonian fluid mechanics 34, 145-180 (1990).

[6] Christelle Combeaud, Yves Demay, and Bruno Vergnes, "Experimental study of the volume defects in polystyrene extrusion," Journal of non-newtonian fluid mechanics 121, 175-185 (2004).

[7] Gareth H McKinley, William P Raiford, Robert A Brown, and Robert C Armstrong, "Nonlinear dynamics of viscoelastic flow in axisymmetric abrupt contractions," Journal of fluid mechanics 223, 411-456 (1991).

[8] Bernard Meulenbroek, Cornelis Storm, Alexander N Morozov, and Wim van Saarloos, "Weakly nonlinear subcritical instability of visco-elastic poiseuille flow," Journal of non-newtonian fluid mechanics 116, 235-268 (2004). 
[9] Bernard Meulenbroek, Cornelis Storm, Volfango Bertola, Christian Wagner, Daniel Bonn, and Wim van Saarloos, "Intrinsic route to melt fracture in polymer extrusion: a weakly nonlinear subcritical instability of viscoelastic poiseuille flow," Physical review letters 90, 024502 (2003).

[10] Helen J Wilson and John M Rallison, "Instability of channel flow of a shear-thinning white-metzner fluid," Journal of non-newtonian fluid mechanics 87, 75-96 (1999).

[11] Helen J Wilson and Vivien Loridan, "Linear instability of a highly shear-thinning fluid in channel flow," Journal of Non-Newtonian Fluid Mechanics 223, 200-208 (2015).

[12] Volfango Bertola, Bernard Meulenbroek, Christian Wagner, Cornelis Storm, Alexander Morozov, Wim van Saarloos, and Daniel Bonn, "Experimental evidence for an intrinsic route to polymer melt fracture phenomena: A nonlinear instability of viscoelastic poiseuille flow," Physical review letters 90, 114502 (2003).

[13] Daniel Bonn, François Ingremeau, Yacine Amarouchene, and Hamid Kellay, "Large velocity fluctuations in smallreynolds-number pipe flow of polymer solutions," Physical Review E 84, 045301 (2011).

[14] L Pan, A Morozov, C Wagner, and PE Arratia, "Nonlinear elastic instability in channel flows at low reynolds numbers," Physical review letters 110, 174502 (2013).

[15] Helen J Wilson, Shear flow instabilities in viscoelastic fluids, Ph.D. thesis, The University of Cambridge (1998).

[16] Hugues Bodiguel, Julien Beaumont, Anaïs Machado, Laetitia Martinie, Hamid Kellay, and Annie Colin, "Flow enhancement due to elastic turbulence in channel flows of shear thinning fluids," Physical review letters $\mathbf{1 1 4}$, 028302 (2015).

[17] RJ Poole, "Elastic instabilities in parallel shear flows of a viscoelastic shear-thinning liquid," Physical Review Fluids 1, 041301 (2016).

[18] Helen J Wilson, "Open mathematical problems regarding non-newtonian fluids," Nonlinearity 25, R45 (2012).

[19] See Supplemental Material at URL for additional details.

[20] Liang Guo, Ralph H Colby, Charles P Lusignan, and Andrew M Howe, "Physical gelation of gelatin studied with rheo-optics," Macromolecules 36, 10009-10020 (2003).

[21] Alexander Morozov and Saverio E Spagnolie, "Introduction to complex fluids," in Complex Fluids in Biological Systems (Springer, 2015) pp. 3-52.

[22] Robert Byron Bird, Robert Calvin Armstrong, Ole Hassager, and Charles F Curtiss, Dynamics of polymeric liquids, Vol. 1 (Wiley New York, 1977).

[23] E Rw Morris, AN Cutler, SB Ross-Murphy, DA Rees, and J Price, "Concentration and shear rate dependence of viscosity in random coil polysaccharide solutions," Carbohydrate polymers 1, 5-21 (1981).

[24] Gérard Iooss and Daniel D Joseph, Elementary stability and bifurcation theory (Springer Science \& Business Media, 2012).

[25] Christelle Combeaud, Etude des instabilités volumiques en extrusion de polystyrène et polypropylène, Ph.D. thesis, École Nationale Supérieure des Mines de Paris (2004).

[26] Christopher JS Petrie and Morton M Denn, "Instabilities in polymer processing," AIChE Journal 22, 209-236 (1976).

[27] RI Tanner, "A theory of die-swell," Journal of Polymer Science Part A-2: Polymer Physics 8, 2067-2078 (1970).

[28] GG Lipscomb, Roland Keunings, and MM Denn, "Implications of boundary singularities in complex geometries," Journal of non-newtonian fluid mechanics 24, 8596 (1987).

[29] Ahmed Allal, A Lavernhe, Bruno Vergnes, and Gérard Marin, "Relationships between molecular structure and sharkskin defect for linear polymers," Journal of nonnewtonian fluid mechanics 134, 127-135 (2006).

[30] Laura Casanellas, Manuel A Alves, Robert J Poole, Sandra Lerouge, and Anke Lindner, "The stabilizing effect of shear thinning on the onset of purely elastic instabilities in serpentine microflows," Soft matter 12, 6167-6175 (2016).

[31] Chaofan Wen, Robert J Poole, Ashley P Willis, and David JC Dennis, "Experimental evidence of symmetrybreaking supercritical transition in pipe flow of shearthinning fluids," Physical Review Fluids 2, 031901 (2017). 